\title{
Automatic High-Spatial-Resolution Nuclear-Magnetic-Resonance Spectroscopy and Imaging System for Rock Cores
}

\author{
Jinhong Chen ${ }^{1}$, Stacey Althaus ${ }^{1}$, Houzhu Zhang ${ }^{1}$ and Mohammed Boudjatit ${ }^{2}$ \\ ${ }^{1}$ Aramco Americas, Houston, Texas, United States, ${ }^{2}$ Saudi Aramco, United States
}

Cores are routinely extracted from oil and gas reservoirs for laboratory study to better understand the conditions and potential productivity, especially at the exploration and appraisal phases in reservoir development. Many essential reservoir properties, such as porosity, permeability, and lithology, among others, are obtained from the core study. Nuclear Magnetic Resonance (NMR) is a physical phenomenon where nuclei in a strong static magnetic field are perturbed by a weak oscillating radio frequency (r.f.) magnetic field and respond by generating an electromagnetic signal in a detection coil. Low field NMR (500 kHz to $2 \mathrm{MHz})$ is a powerful logging technology for estimating fluid content and characterizing fluid transport properties in a reservoir $[1,2]$ and may be the most important logging method for unconventional reservoirs. Low field NMR (1 MHz to $30 \mathrm{MHz}$ ) has also been widely used in laboratories for core plug analysis of conventional and unconventional rocks to provide accurate petrophysical measurements and for calibration of borehole log data [3, 4]. However, current laboratory NMR technology developed to detect fluids in rock samples is mainly for samples shorter than the detection coil with some fundamental limiting factors: first, the spatial resolution of acquired data is limited by the finite r.f. coil length. High-spatial-resolution NMR (HSR-NMR) results are often desired because the reservoir, and therefore the cores, can be significantly laminated with layers much thinner than the coil aperture. Second, the end effect of the finite length r.f. coil causes inaccuracy of the measurement. For a finite coil, the r.f. field is not homogeneous, especially at the two ends of the coil. The measured signal includes signal from sample at the two ends of the r.f. coil, especially for samples longer than the coil length, in addition to the homogeneous part of the sample within the coil.

Here we show a method that overcomes these limits to acquire HSR-NMR images or spectroscopy with resolution much higher than the coil aperture on samples longer than the coil. The HSR-NMR method acquires NMR data in synchronization with stepwise movement of the long sample through the r.f. coil with step increments equal to the desired spatial resolution. An inversion process was applied on the acquired data to obtain HSR-NMR spectroscopy and/or images. This method

- provides a measurement of fluid content and images with spatial resolution that is not limited by the length of the r.f. coil used in the instrument. This can be especially useful in laboratory applications for rock samples with thin laminations.

- accounts for and, thus, is not limited by, the end effects of the finite length of the $r f$ coil on the accuracy of the measurement.

- delivers quantitative images or measurements for material with a very short transverse relaxation time.

An automatic laboratory NMR spectrometer was built to implement this method for rock plugs. Figure 1 shows the acquired HSR-NMR spectroscopy using the current method (c) in comparison with the traditional pulse-field-gradient (PFG) based MRI method (b) on a stacked tri-rock sample. The fluid signal in the shale sample, with very short transverse relaxation time, were acquired using this new method but lost using the traditional method.

The HSR-NMR method was also implemented to acquire HSR images and spectroscopy on preserved whole cores of 4-inch diameter. Figure 2 shows an example of the acquired HSR fluid distribution and NMR T2 spectra on a preserved whole core. The acquired fluid distribution correlates well with the 
variations of organic carbon in the cores. Further experiments showed that the acquired fluid content from this method matched well with the fluid using industry standard GRI method [5].

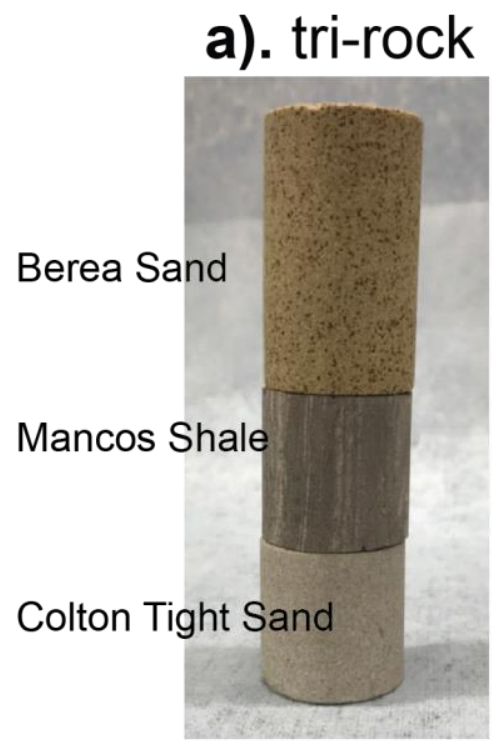

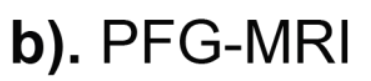

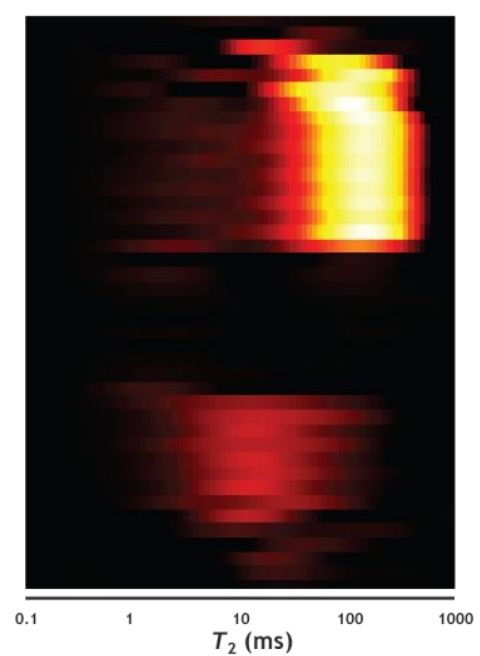

\section{c). HSR-NMR}

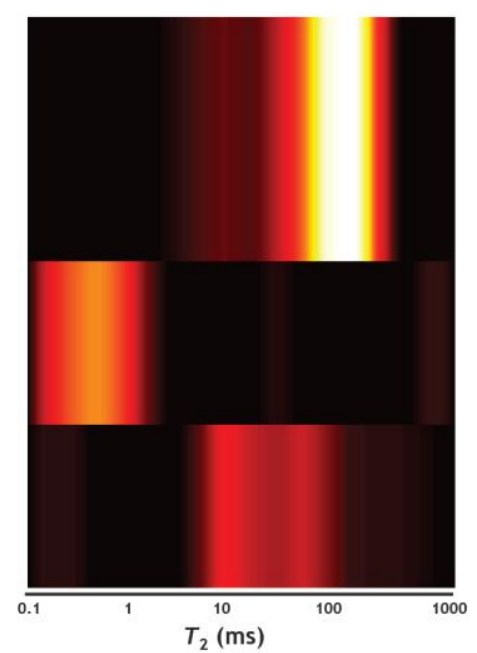

Figure 1. Figure 1. Comparison of HSR-NMR spectroscopy acquired using current HSR-NMR method (c) with traditional MRI based method (b) on a stacked tri-rock sample. Fluid signals in shales with short relaxation time were retained in current method but lost in tradition method.
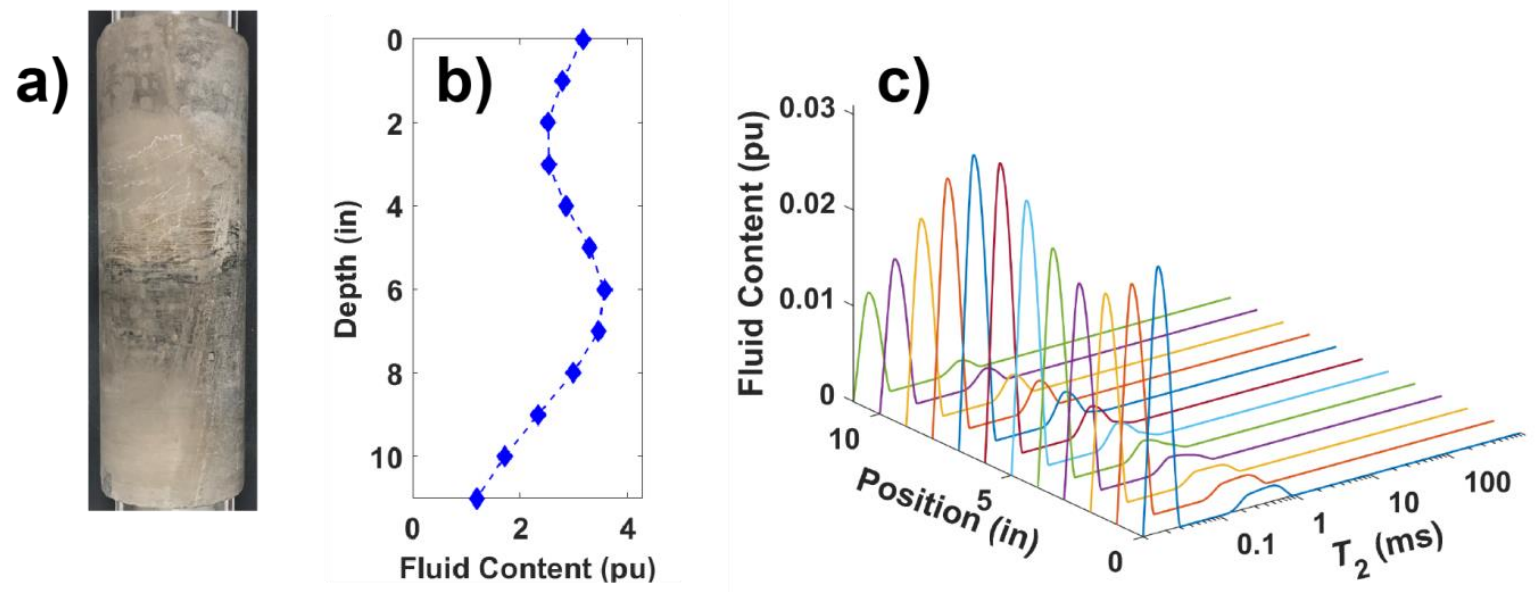

Figure 2. Figure 2. Acquired HSR fluid distribution (b) and NMR T2 spectra (c) in a preserved source rock core.

\section{References}

Chang D, Vinegar HJ, Morriss C, Straley C. Effective Porosity, Producible Fluid And Permeability In Carbonates From Nmr Logging. SPWLA 35th Annual Logging Symposium. Tulsa, Oklahoma: Society of Petrophysicists and Well-Log Analysts; 1994:21.

[2] Dunn KJ, Bergman DJ, LaTorraca GA. Nuclear Magnetic Resonance: Petrophysical and Logging Applications. Elsevier Science; 2002. 
[3] Timur A. Pulsed Nuclear Magnetic Resonance Studies of Porosity, Movable Fluid, and Permeability of Sandstones. 1969.

[4] Chen J-H, Zhang J, Jin G, Quinn T, Frost E, Chen J. Capillary Condensation And NMR Relaxation Time In Unconventional Shale Hydrocarbon Resources. Society of Petrophysicists and Well-Log Analysts; 2012.

[5] Luffel DL, Guidry FK. New Core Analysis Methods for Measuring Reservoir Rock Properties of Devonian Shale. 1992. 